\title{
二苯甲酮腙与芳基氯化物及芳基硼酸的碳氮键偶联反应
}

\author{
姚丹丹张金利* 徐 亮* \\ (石河子大学化学化工学院 新疆兵团化工绿色过程重点实验室 新疆石河子 832003)
}

\begin{abstract}
摘要 钯催化的芳基卤化物与 $\mathrm{N}$-亲核试剂的 Buchwald-Hartwig 反应以及铜催化的芳基硼酸与 $\mathrm{N}$-亲核试剂的 Chan-Evans-Lam 反应都是构建 $\mathrm{C}-\mathrm{N}$ 键的有效方法. 在醋酸钯/叔丁醇体系或醋酸铜/二氯甲烷体系中, 芳基氯化物或芳 基硼酸分别与二苯甲酮腙反应构筑 $\mathrm{C}-\mathrm{N}$ 键，生成相应的芳基腙，芳基腙水解之后可得到芳基肼. 该类反应提供了一条 利用低毒性的化学试剂合成芳基肼的间接路径.
\end{abstract}

关键词 Buchwald-Hartwig 反应; Chan-Evans-Lam 反应; 芳基硼酸; 二苯甲酮腙

\section{C-N Coupling Reactions between Benzophenone Hydrazone and Aryl Chlorides and Boronic Acids}

\author{
Yao, Dandan Zhang, Jinli* Xu, Liang* \\ (Key Laboratory for Green Processing of Chemical Engineering of Xinjiang Bingtuan, School of Chemistry and Chemical \\ Engineering, Shihezi University, Shihezi, Xinjiang 832003)
}

\begin{abstract}
Palladium catalyzed Buchwald-Hartwig reactions between aryl halides and $N$-nucleophiles, and the copper catalyzed Chan-Evans-Lam reactions between aryl boronic acids and $N$-nucleophiles are all effective methods for constructing $\mathrm{C}-\mathrm{N}$ bonds. Herein, under palladium acetate/tert-butanol system or copper acetate/dichloromethane system, benzophenone hydrazone can react with aryl chlorides and aryl boronic acids to afford corresponding aryl hydrazones, respectively. The obtained products are easily hydrolyzed to form aryl hydrazines, thus providing an indirect pathway to access aryl hydrazines from relatively less toxic reagents.
\end{abstract}

Keywords Buchwald-Hartwig reaction; Chan-Evans-Lam reaction; aryl boronic acid; benzophenone hydrazone

芳基肼以及芳基腙是重要的有机合成中间体，被广

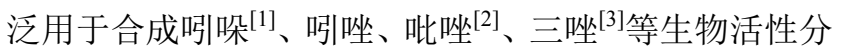
子中常见的含氮杂环 ${ }^{[4]}$. 此外, 多种药物分子中也含有 芳基肼结构单元, 如治疗高血压的肼屈嗪和抗炎药保泰 松等. 因此，这类中间体制备方法的开创和改进一直是 有机合成研究中的重要内容之一.

传统的芳基肼的制备方法中, 将苯胺氧化成相应的 重氮盐, 再将重氮盐还原可得到芳基肼, 常用的还原剂 包括锡(II)盐和亚硫酸盐等. 该方法反应条件较为苛刻, 官能团耐受性差, 会产生大量废盐. 随着过渡金属催化 的交叉偶联的发展, 尤其是各种碳氮键偶联反应的开发 以及推广利用, 一系列合成芳基肼的替代方案被开发出 来, 主要包括: (1)钯催化的 Buchwald-Hartwig 型反应 ${ }^{[5]}$, 如钯催化的(拟)卤代芳烃与二苯甲酮腙 ${ }^{[6]}$ 或保护的酰肼
的 $N$-芳基化反应 ${ }^{[7]}$ 以及钯催化下(拟)卤代芳烃与肼的直 接碳氮偶联反应 ${ }^{[8]} ;$, (2)铜催化的 Ullmann 型反应 ${ }^{[9]}$, 如铜 催化的(拟)卤代芳烃与酰朋 ${ }^{[10]}$ 、苯肼或水合肤 ${ }^{[11]}$ 的芳基 化反应. 其中, 以酰肼、腙为原料时, 反应结束后, 得到 的碳氮键偶联产物为芳基肼衍生物, 后续的转化将得到 未保护的芳基肼, 这样可以避免使用毒性较大的水合肼 作为起始原料, 提高反应过程的安全性.

在二苯甲酮腙的 $N$-芳基化反应中, 通常使用反应 活性较高的溴代芳烃 ${ }^{[\mathrm{ad}, 4 \mathrm{c}, 4 \mathrm{f}, 6 \mathrm{~b}, 10 \mathrm{c}, 10 \mathrm{~d}, 12]}$ 及芳基碘 ${ }^{[10,11]}$ 作为 起始原料, 以更加廉价的氯代芳烃为原料的反应仅有零 星的报道 ${ }^{[6 c, 13]}$. 另一方面，以芳基硼酸作为反应原料的 Chan-Evans-Lam 反应 ${ }^{[14]}$ 可以实现碳氮键的氧化偶联, 该类反应大多对环境中的水和氧不敏感，反应条件简 单，操作方便，近些年来获得了较好的发展. 多种多样

*Corresponding authors. E-mail: zhangjinli@tju.edu.cn; xuliang4423@shzu.edu.cn

Received December 26, 2019; revised February 21, 2020; published online March 6, 2020.

Project supported by the National Natural Science Foundation of China (Nos. 21603150, 21963010).

国家自然科学基金(Nos. 21603150, 21963010)资助项目. 
的 $N$-亲核试剂都可与芳基嗍酸发生 Chan-Evans-Lam 反 应以构筑碳氮键, 但目前仍没有将二苯甲酮腙用于该类 反应的报道. 如果在适当的反应条件下, 实现氯代芳烃 以及芳基硼酸与二苯甲酩腙的快速高效偶联, 将拓宽芳 基腙以及芳基肼来源，降低该类反应的成本，增加其实 用价值. 基于本课题组先前的工作基础 ${ }^{[15]}$, 本工作将氯 化芳烃、芳基硼酸与二苯甲酮腙的碳氮键偶联反应分别 在金属钯和铜的催化下完成，得到一系列相应的芳基腙 化合物。

\section{1 结果与讨论}

\section{1 氯代芳烃与二苯甲酮腙的反应}

在 Buchwald-Hartwig 反应中, 大位阻单齿膦配体的 使用可以提高反应的效率, 促使氯代芳烃参与碳氮键偶 联反应 ${ }^{[16]}$. 我们设想, 在钯催化的氯代芳烃与二苯甲酮 腙的反应中使用大位阻单齿膦配体, 有可能得到预期的 芳基腙类产物. 基于以上设想, 首先研究了钯催化下氯 苯 $(1 \mathrm{a})$ 与二苯甲酮腙 $(2 \mathrm{a})$ 的偶联反应. 以乙醇为溶剂, 以氢氧化钠为碱, 两种反应物在 $0.5 \mathrm{~mol} \%$ 钯催化剂和 1 mol\% MePhos 促进下 $90{ }^{\circ} \mathrm{C}$ 下反应 $5 \mathrm{~h}$, 以 $67 \%$ 的产率得 到目标产物 3a(表 1, Entry 1).

条件篮选发现反应溶剂对偶联反应产生非常大的 影响. 当以二甲亚砜(DMSO)、四氢呋喃(THF)、二氧六 环(1,4-dioxane)作溶剂时, 无法得到目标产物, 而使用 叔丁醇可得到比乙醇更好的反应收率 $(82 \%$, Entry 2$)$. 当 使用 $\mathrm{Pd}\left(\mathrm{PPh}_{3}\right)_{4}$ 作为钯催化剂前体时, 反应产率仅为 $19 \%$. 产率的下降可能是由于 $\mathrm{PPh}_{3}$ 与 $\mathbf{L}_{\mathbf{1}}$ 竞争配位引起 的. 对 Buchwald 型配体的篎选发现, 使用 tBuDavePhos $\left(\mathbf{L}_{2}\right)$ 可得到更高的收率(Entry 9). 碱是影响收率的另一 重要因素. 使用无机强碱 $\mathrm{NaOH}$ 时得到 $87 \%$ 的收率, 使 用无机弱碱 $\mathrm{Na}_{2} \mathrm{CO}_{3}$ 时收率明显下降(Entry 14), 而使用 有机碱三乙胺(TEA)和 1,8-二氮杂二环十一碳-7-烯 (DBU) (Entries 12、13)时反应效果明显比无机碱差. 最 后，反应温度降低时反应产率下降(Entry 15). 因此，最 终确认最优反应条件为: $\operatorname{Pd}(\mathrm{OAc})_{2}$ 为催化剂, $\mathbf{L}_{\mathbf{2}}$ 为配体, $\mathrm{NaOH}$ 为碱，两种原料于 $90{ }^{\circ} \mathrm{C}$ 的叔丁醇中反应 $5 \mathrm{~h}$.

对上述最优条件的底物适用性进行了考察. 选取不 同的芳基氯化物与二苯甲酮腙反应，结果见表 2 . 从电 性的角度看, 无论氯代芳烃上连有供电子基团还是吸电 子基团，与二苯甲酮腙的偶联反应均可发生，且大多数 能以 $60 \% \sim 90 \%$ 的产率得到相应的目标产物. 甲氧基 (3b , 88\%；3c，90\%)、三氟甲基(3e，65\%；3m，55\%；3o, 69\%)、酮羰基(3f, 79\%; 3g, 85\%)、酯基(31, 41\%)、硝基 $(3 \mathrm{~h}, 78 \%)$ 均可与该反应体系兼容. 从位阻的角度看, 2甲氧基氯苯与 3-甲氧基氯苯和 4-三氟甲基氯苯与 2-
表 1 氯苯与二苯甲酮腙的反应优化 ${ }^{a}$

Table 1 Optimization of reactions between aryl chlorides and benzophenone hydrazones

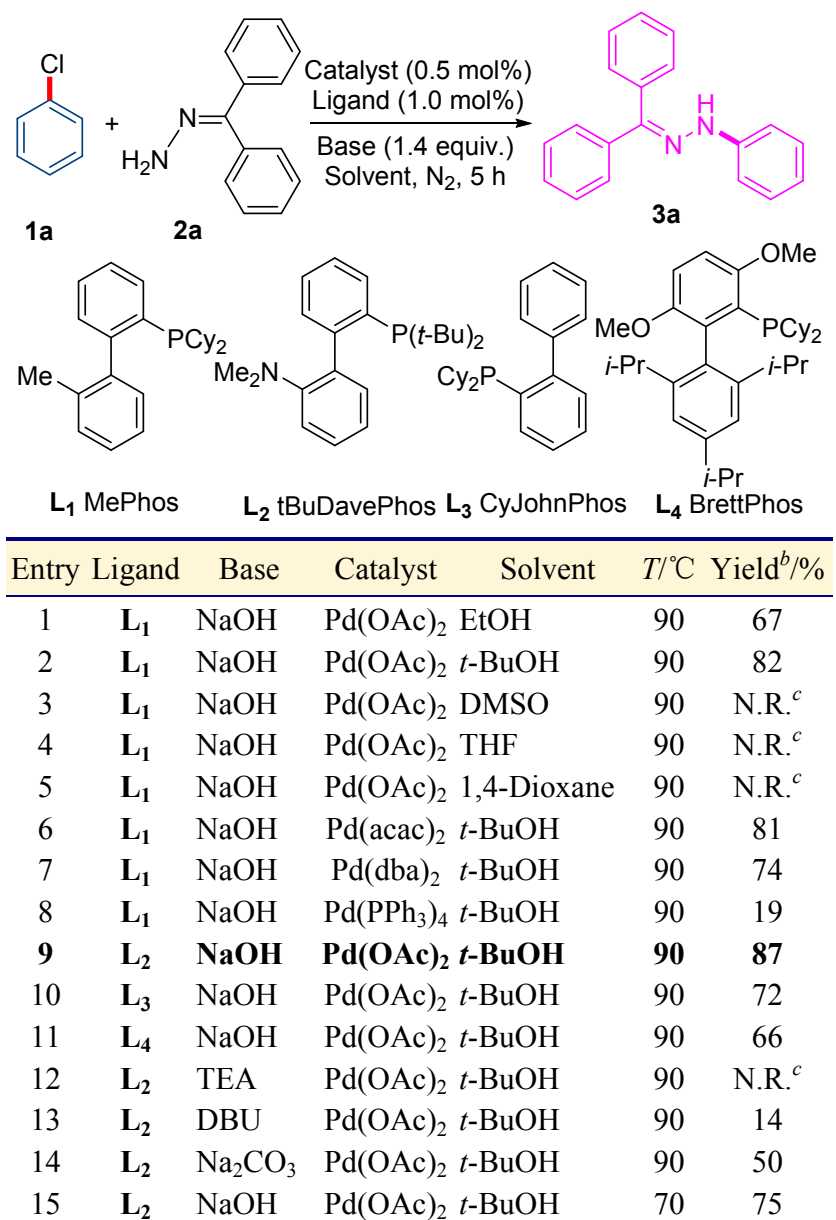

${ }^{a}$ Reaction conditions: 1a $(0.30 \mathrm{mmol}, 1.0$ equiv.), $2 \mathbf{a}(0.45 \mathrm{mmol}, 1.5$ equiv. $)$, catalyst $(0.0015 \mathrm{mmol}, 0.5 \mathrm{~mol} \%)$, ligand $(0.003 \mathrm{mmol}, 1 \mathrm{~mol} \%)$, base $(0.42$ mmol, 1.4 equiv.), solvent $(0.30 \mathrm{~mL}), 5 \mathrm{~h} .{ }^{b}$ Isolated yields. ${ }^{c}$ No reaction.

三氟甲基氯苯发生反应后得到相应的产物的产率相近， 说明邻位取代基的位阻对反应性并无明显的影响.

\section{2 芳基硼酸与二苯甲酮腙的反应}

在完成钯催化的氯代芳烃与二苯甲酮腙的反应之 后，尝试了芳基硼酸与二苯甲酮腙的碳氮偶联反应 $($ 表 $3)$. 将 1.0 equiv. 的苯硼酸(4a)、1.5 equiv.的二苯甲酮腙、 1.5 equiv. 的醋酸铜和 2.0 equiv. 的吡啶氮氧化物混合于 二氯甲烷溶剂中, 室温搅拌 $24 \mathrm{~h}$, 以 $63 \%$ 的产率得到目 标产物. 使用其他铜盐，反应产率将明显下降(Entries 2, 3). 当反应体系中加入不同的双齿配体时，得到 50\% $76 \%$ 的产率，其中四甲基乙二胺(TMEDA，Entry 5)以及 氨基酸类配体(Entries 6,7)将降低反应的产率，而草酰 二胺类配体 $\mathbf{L}_{\mathbf{A}}$ (Entry 8) 的使用将使产率提升至 $76 \%$. 在此基础上，反应体系中加入 $0.1 \mathrm{~g} 4 \AA$ 分子篎，将使反 应产率小幅提升至 $80 \%$ (Entry 10). 延长反应时间至 36 或 $48 \mathrm{~h}$ 并不会提高反应的产率. 当将醋酸铜用量降至 
表 2 氯苯与二苯甲酮腙反应的底物适用范围

Table 2 Substrate scope of the reactions between aryl chlorides and benzophenone hydrazones

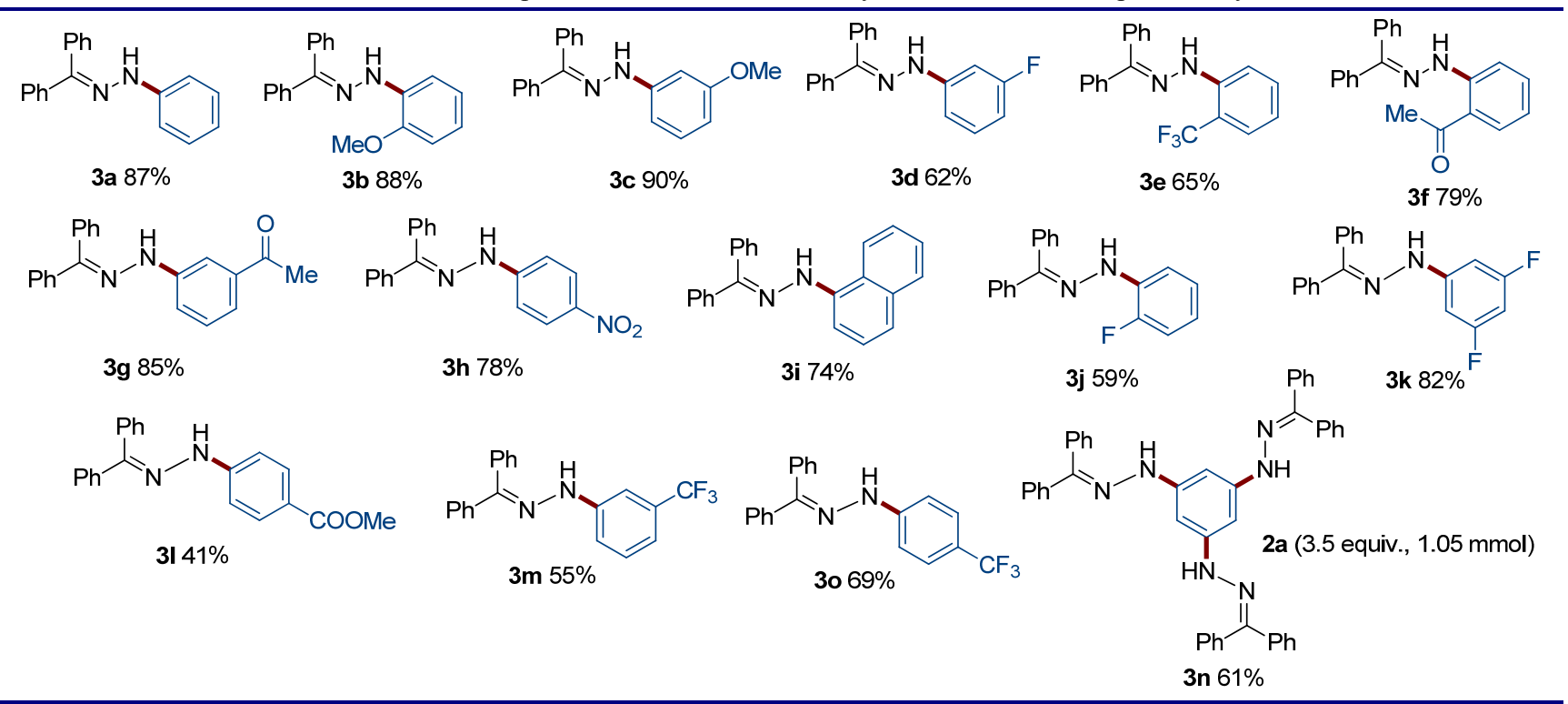

表 3 芳基硼酸与二苯甲酮腙的反应优化 ${ }^{a}$

Table 3 Optimization of reactions between aryl boronic acids and benzophenone hydrazones

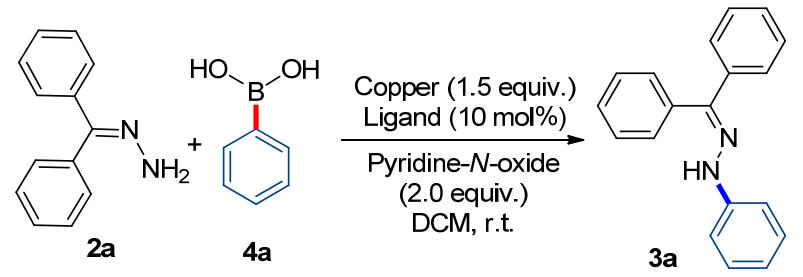<smiles>Cc1cc(C)c(NC(=O)C(=O)Nc2c(C)cc(C)cc2C)c(C)c1</smiles>

$\mathrm{L}_{\mathrm{A}} N^{1}$-benzyl- $N^{2}$-phenyloxalamide $\mathrm{L}_{\mathrm{B}} N^{1}, N^{2}$-dimesityloxalamide

\begin{tabular}{clccc}
\hline Entry & Copper & Ligand & Time/h & Yield $^{b} \%$ \\
\hline 1 & $\mathrm{Cu}(\mathrm{OAc})_{2}$ & - & 24 & 63 \\
2 & $\mathrm{CuTC}$ & - & 24 & 53 \\
3 & $\mathrm{Cu}(\mathrm{OTf})_{2}$ & - & 24 & 32 \\
4 & $\mathrm{Cu}(\mathrm{OAc})_{2}$ & Salicylamide & 24 & 52 \\
5 & $\mathrm{Cu}(\mathrm{OAc})_{2}$ & TMEDA & 24 & 50 \\
6 & $\mathrm{Cu}(\mathrm{OAc})_{2}$ & $\beta$-Alanine & 24 & 51 \\
7 & $\mathrm{Cu}(\mathrm{OAc})_{2}$ & $L$-Alanine & 24 & 64 \\
8 & $\mathrm{Cu}(\mathrm{OAc})_{2}$ & $\mathbf{L}_{\mathbf{A}}$ & 24 & 76 \\
9 & $\mathrm{Cu}(\mathrm{OAc})_{2}$ & $\mathbf{L}_{\mathbf{B}}$ & 24 & 70 \\
$\mathbf{1 0}$ & $\mathbf{C u}(\mathrm{OAc})_{2}$ & $\mathbf{L}_{\mathbf{A}}$ & $\mathbf{2 4}$ & $\mathbf{8 0}^{c}$ \\
11 & $\mathrm{Cu}(\mathrm{OAc})_{2}$ & $\mathbf{L}_{\mathbf{A}}$ & 36 & $75^{c}$ \\
12 & $\mathrm{Cu}(\mathrm{OAc})_{2}$ & $\mathbf{L}_{\mathbf{A}}$ & 48 & $77^{c}$ \\
13 & $\mathrm{Cu}(\mathrm{OAc})_{2}$ & $\mathbf{L}_{\mathbf{A}}$ & 24 & $71^{c, d}$ \\
\hline
\end{tabular}

${ }^{a}$ Reaction conditions: $\mathbf{4 a}(0.15 \mathrm{mmol}, 1.0$ equiv. $), \mathbf{2 a}(0.225 \mathrm{mmol}, 1.5$ equiv. $)$, $\mathrm{Cu}$ salts $(0.225 \mathrm{mmol}, 1.5$ equiv.), ligand $(0.015 \mathrm{mmol}, 10 \mathrm{~mol} \%)$, oxidant $\left(0.30 \mathrm{mmol}, 2.0\right.$ equiv.), solvent $(1.0 \mathrm{~mL}), 24 \mathrm{~h} .{ }^{b}$ Isolated yield. ${ }^{c} 4 \AA \mathrm{MS}(100$ $\mathrm{mg}){ }^{d}$ Catalyst (0.0225 mmol, $\left.15 \mathrm{~mol} \%\right)$, ligand ( $\left.0.045 \mathrm{mmol}, 30 \mathrm{~mol} \%\right)$.

4a 的 0.15 equiv. 时, 反应产率下降至 $71 \%$ (Entry 13). 根
据以上的结果分析，最终确定了最优的反应条件，即： 以二氯甲烷作为溶剂, 醋酸铜作为反应的促进剂, 以 $N^{1}$-苄基- $N^{2}$-苯基草酰二胺为配体，吡啶氮氧化物作为氧 化剂, $4 \AA$ 分子篮为添加剂, 实现芳基硼酸与二苯甲酮腙 的碳氮偶联反应.

在笁选出的最优的反应条件下，对二苯甲酮腙与芳 基硼酸的反应进行了底物的拓展，研究了含有不同取代 基的芳基硼酸与二苯甲酮腙的反应，结果如表 4 所示. 相对于氯代芳烃的反应，芳基硼酸的反应产率普遍较 低，通常可以以中等到良好的产率( $42 \% \sim 75 \%)$ 得到目 标产物. 当芳基嗍酸连接有氟、氯和溴等卤素原子时 $(\mathbf{5 e}$, 5f，3d), 反应可正常进行, 说明该反应可与上述的钯催 化卤苯的碳氮键偶联相互补充, 也有利于所得到目标分 子的后续修饰和官能团化.

\section{3 二苯甲酮一苯腙水解制备苯肼}

上述反应所得到的苯腙可在盐酸与乙醇的体系下 进行水解反应(Eq. 1), 常温下搅拌 $24 \mathrm{~h}$, 反应后无需进 行柱层析分离, 以少量的二氯甲烷进行 3 4 次的洗脱, 即可得到白色的芳基肼盐酸盐产物, 反应产率 56\%.

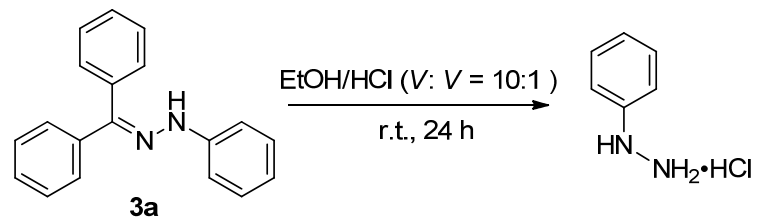

\section{2 结论}

报道了两种二苯甲酮腙的 $N$-芳基化反应，以制备 二苯甲酮一芳基腙类化合物. 两种方法分别为钯催化下 
表 4 芳基硼酸与二苯甲酮腙反应的底物适用范围

Table 4 Substrate scope of reactions between aryl boronic acids and benzophenone hydrazones
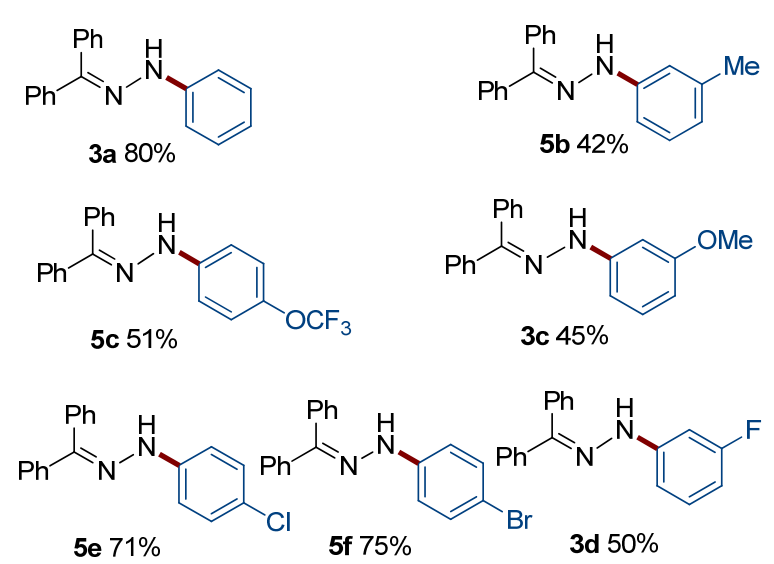

与氯代芳烃的 Buchwald-Hartwig 反应(反应产率 $41 \%$ $90 \%$ ) 以及铜促进的与芳基硼酸的 Chan-Evans-Lam 反应 (反应产率 $45 \% \sim 75 \%$ ). 反应所得的芳基腙化合物可方 便地水解为芳基肼, 避免了水合肼在芳基肼合成中的使 用，为该类化合物的合成提供了一条相对安全、低毒的 路径.

\section{3 实验部分}

\section{1 仪器与试剂}

${ }^{1} \mathrm{H}$ NMR (400 MHz) 和 ${ }^{13} \mathrm{C}$ NMR (101 MHz)采用 Bruker Ascend-400 MHz 核磁共振仪测定; 红外测试使 用 Thermo Fisher Nicolet 6700 FT-IR 红外光谱仪利用 ATR (Attenuated Total Reflectance)方法测试. 柱层析使 用青岛海洋化工生产的 $200 \sim 300$ 目硅胶, 实验所用溶 剂和试剂(包括原料 2a)均未经后续处理纯化.

\section{2 实验方法}

\subsection{1 氯代芳烃的反应}

依次向 $25 \mathrm{~mL}$ 的反应管中加入 $0.30 \mathrm{mmol}$ 芳基氯化 物, $0.45 \mathrm{mmol}$ 二苯甲酮腙, $0.0015 \mathrm{mmol}$ 醋酸钯, 0.003 $\mathrm{mmol}$ 配体和 $0.42 \mathrm{mmol}$ 氢氧化钠, 以氮气置换反应管内 气体. 在氮气保护条件下加入 $0.30 \mathrm{~mL}$ 叔丁醇, 在 $90{ }^{\circ} \mathrm{C}$ 油浴中反应 $5 \mathrm{~h}$, 冷却至室温. 反应液经过抽滤、乙酸乙 酯洗涤, 滤液转移到 $50 \mathrm{~mL}$ 的鸡心瓶中, 蒸除溶剂. 粗 产物经柱层析纯化 [洗脱剂: 石油醚/乙酸乙酯 $(V: V=$ $100: 1)$ ]得到相应的产物.

二苯甲酮苯腙(3a): $71 \mathrm{mg}$, 黄色固体, 产率 $87 \%$. m.p. $135 \sim 136{ }^{\circ} \mathrm{C}$ (lit. ${ }^{[12 \mathrm{e}]}$ m.p. $134 \sim 135{ }^{\circ} \mathrm{C}$ ); ${ }^{1} \mathrm{H}$ NMR $\left(400 \mathrm{MHz}, \mathrm{CDCl}_{3}\right) \delta: 7.63 \sim 7.54(\mathrm{~m}, 4 \mathrm{H}), 7.54 \sim 7.45(\mathrm{~m}$, $2 \mathrm{H}), 7.35 \sim 7.29(\mathrm{~m}, 4 \mathrm{H}), 7.28 \sim 7.21(\mathrm{~m}, 3 \mathrm{H}), 7.08(\mathrm{dd}$, $J=8.5,0.9 \mathrm{~Hz}, 2 \mathrm{H}), 6.84(\mathrm{t}, J=7.3 \mathrm{~Hz}, 1 \mathrm{H}) ;{ }^{13} \mathrm{C} \mathrm{NMR}$ $\left(101 \mathrm{MHz}, \mathrm{CDCl}_{3}\right) \delta: 144.6,144.2,138.4,132.8,129.7$, $129.3,129.2,129.2,128.2,128.0,126.5,120.1,113.0$.

二苯甲酮-2-甲氧基苯腙(3b): $80 \mathrm{mg}$, 黄色固体，产 率 88\%. m.p. $154 \sim 156{ }^{\circ} \mathrm{C}$ (lit. ${ }^{[12 \mathrm{e}]}$ m.p. $155 \sim 156{ }^{\circ} \mathrm{C}$ ); ${ }^{1} \mathrm{H}$ NMR (400 MHz, $\left.\mathrm{CDCl}_{3}\right) \delta: 7.99$ (s, 1H), 7.68 (d, $J=$ $8.0 \mathrm{~Hz}, 1 \mathrm{H}), 7.62 \sim 7.49(\mathrm{~m}, 5 \mathrm{H}), 7.37 \sim 7.27(\mathrm{~m}, 5 \mathrm{H}), 6.98$ $(\mathrm{t}, J=8.0 \mathrm{~Hz}, 1 \mathrm{H}), 6.82 \sim 6.75(\mathrm{~m}, 2 \mathrm{H}), 3.68(\mathrm{~s}, 3 \mathrm{H}) ;{ }^{13} \mathrm{C}$ NMR (101 MHz, $\left.\mathrm{CDCl}_{3}\right) \delta: 145.4,144.8,138.6,134.3$, $133.1,129.6,129.1,128.2,127.9,126.6,121.6,119.2$, 112.4, 110.1, 55.6 .

二苯甲酮-3-甲氧基苯腙(3c)：82 mg, 黄色固体，产 率 90\%. m.p. 102 103 ${ }^{\circ} \mathrm{C}$ (lit. ${ }^{[12 \mathrm{e}]}$ m.p. $102 \sim 103{ }^{\circ} \mathrm{C}$ ); ${ }^{1} \mathrm{H}$ NMR $\left(400 \mathrm{MHz}, \mathrm{CDCl}_{3}\right) \delta: 7.59 \sim 7.48(\mathrm{~m}, 6 \mathrm{H})$, $7.33 \sim 7.27(\mathrm{~m}, 5 \mathrm{H}), 7.12(\mathrm{t}, J=8.0 \mathrm{~Hz}, 1 \mathrm{H}), 6.78(\mathrm{~s}, 1 \mathrm{H})$, $6.55(\mathrm{~d}, J=8.0 \mathrm{~Hz}, 1 \mathrm{H}), 6.41(\mathrm{~d}, J=8.0 \mathrm{~Hz}, 1 \mathrm{H}), 3.80$ (s, $3 \mathrm{H}) ;{ }^{13} \mathrm{C} \mathrm{NMR}\left(101 \mathrm{MHz}, \mathrm{CDCl}_{3}\right) \delta: 160.9,146.0,144.3$, $138.3,132.8,130.0,129.7,129.3,129.2,128.2,128.1$, 126.5, 105.7, 105.7, 98.8, 55.3.

二苯甲酮-3-氟苯腙(3d): $54 \mathrm{mg}$, 黄色油状物, 产率 $62 \%$. ${ }^{1} \mathrm{H}$ NMR (400 MHz, $\left.\mathrm{CDCl}_{3}\right) \delta: 7.61 \sim 7.51(\mathrm{~m}, 6 \mathrm{H})$, 7.33 (d, $J=8.0 \mathrm{~Hz}, 5 \mathrm{H}), 7.14$ (q, $J=12.0 \mathrm{~Hz}, 1 \mathrm{H}), 6.94$ (d, $J=12.0 \mathrm{~Hz}, 1 \mathrm{H}), 6.69(\mathrm{~d}, J=8.0 \mathrm{~Hz}, 1 \mathrm{H}), 6.52(\mathrm{t}, J=8.0$ $\mathrm{Hz}, 1 \mathrm{H}) ;{ }^{13} \mathrm{C} \mathrm{NMR}\left(101 \mathrm{MHz}, \mathrm{CDCl}_{3}\right) \delta: 164.0(\mathrm{~d}, J=243$ $\mathrm{Hz}), 146.4$ (d, $J=11 \mathrm{~Hz}), 145.2,138.1,132.6,130.3$ (d, $J=10 \mathrm{~Hz}), 129.8,129.5,129.1,128.4,128.3,126.7,108.6$ (d, $J=2.0 \mathrm{~Hz}), 106.5$ (d, $J=22 \mathrm{~Hz}), 100.2$ (d, $J=26 \mathrm{~Hz})$; ${ }^{19} \mathrm{~F}$ NMR (376 MHz, $\mathrm{CDCl}_{3}$ ) $\delta$ : -112.32 ; IR (ATR) $v$ : 3451, 1611, 1274, 1258, 1074, 759, $698 \mathrm{~cm}^{-1}$; HRMS (ESI) calcd for $\mathrm{C}_{19} \mathrm{H}_{16} \mathrm{FN}_{2}[\mathrm{M}+\mathrm{H}]^{+}$291.1292, found 291.1295 .

二苯甲酮-2-三氟甲基苯腙(3e)：66 mg，黄色油状 物, 产率 $65 \% .{ }^{1} \mathrm{H}$ NMR $\left(400 \mathrm{MHz}, \mathrm{CDCl}_{3}\right) \delta: 8.07(\mathrm{~s}$, $1 \mathrm{H}), 7.93(\mathrm{~d}, J=8.0 \mathrm{~Hz}, 1 \mathrm{H}), 7.65 \sim 7.47(\mathrm{~m}, 6 \mathrm{H}), 7.40$ (d, $J=8.0 \mathrm{~Hz}, 1 \mathrm{H}), 7.35 \sim 7.32(\mathrm{~m}, 5 \mathrm{H}), 6.86(\mathrm{~d}, J=8.0 \mathrm{~Hz}$, $1 \mathrm{H}) ;{ }^{13} \mathrm{C}$ NMR (101 MHz, DMSO- $\left.d_{6}\right) \delta: 148.2,141.9$, $137.5,134.5,132.1,130.4,130.3,129.4,129.0,128.9$, 126.9, 126.6 (q, $J=6 \mathrm{~Hz}), 124.9$ (q, $J=270 \mathrm{~Hz}$ ), 119.8, 114.6, 111.4 (q, $J=29 \mathrm{~Hz}$ ); ${ }^{19} \mathrm{~F}$ NMR (376 MHz, DMSO$\left.d_{6}\right) \delta:-60.70$; IR (ATR) $v: 3374,1516,1321,1303,1166$, $788,754 \mathrm{~cm}^{-1}$; HRMS (ESI) calcd for $\mathrm{C}_{20} \mathrm{H}_{16} \mathrm{~F}_{3} \mathrm{~N}_{2}[\mathrm{M}+$ $\mathrm{H}]^{+}$341.1260, found 341.1263.

二苯甲酮-2-乙酰基苯腙(3f): $74 \mathrm{mg}$, 黄色油状物, 产率 79\%. ${ }^{1} \mathrm{H}$ NMR $\left(400 \mathrm{MHz}, \mathrm{CDCl}_{3}\right) \delta: 7.73(\mathrm{~d}, J=8.0$ $\mathrm{Hz}, 2 \mathrm{H}), 7.40$ (q, $J=8.0 \mathrm{~Hz}, 6 \mathrm{H}), 7.34 \sim 7.27$ (m, 4H), $7.23 \sim 7.12(\mathrm{~m}, 3 \mathrm{H}), 2.33(\mathrm{~s}, 3 \mathrm{H}) ;{ }^{13} \mathrm{C} \mathrm{NMR}(101 \mathrm{MHz}$, 
$\left.\mathrm{CDCl}_{3}\right) \delta: 158.8,158.5,139.2,137.9,135.2,132.1,130.0$, $129.9,129.7,129.6,129.0,128.9,128.6,128.2,128.1$, 126.8, 19.9; IR (ATR) v: 3431, 1701, 1612, 1359, 1301, 1099, 792, $735 \mathrm{~cm}^{-1}$; HRMS (ESI) calcd for $\mathrm{C}_{21} \mathrm{H}_{19} \mathrm{~N}_{2} \mathrm{O}$ $(\mathrm{M}+\mathrm{H})^{+}$315.1492, found 315.1495.

二苯甲酮-4-乙酰基苯腙(3g): $80 \mathrm{mg}$, 黄色油状物, 产率 85\%. ${ }^{1} \mathrm{H}$ NMR $\left(400 \mathrm{MHz}, \mathrm{CDCl}_{3}\right) \delta: 7.89(\mathrm{~d}, J=8.0$ $\mathrm{Hz}, 2 \mathrm{H}), 7.74(\mathrm{~s}, 1 \mathrm{H}), 7.63 \sim 7.55(\mathrm{~m}, 5 \mathrm{H}), 7.34$ (d, $J=8.0$ $\mathrm{Hz}, 5 \mathrm{H}), 7.09$ (d, $J=8.0 \mathrm{~Hz}, 2 \mathrm{H}), 2.54(\mathrm{~s}, 3 \mathrm{H}) ;{ }^{13} \mathrm{C} \mathrm{NMR}$ $\left(101 \mathrm{MHz}, \mathrm{CDCl}_{3}\right) \delta: 196.6,148.3,146.9,130.6,130.1$, $129.8,129.6,129.3,129.0,128.7,128.3,128.3,126.8$, 112.2, 26.2; IR (ATR) $v: 3445,1705,1609,1347,1297$, $1099,847,789 \mathrm{~cm}^{-1}$; HRMS (ESI) calcd for $\mathrm{C}_{21} \mathrm{H}_{19} \mathrm{~N}_{2} \mathrm{O}$ $[\mathrm{M}+\mathrm{H}]^{+}$315.1492, found 315.1495.

二苯甲酮-4-硝基苯腙 $(\mathbf{3 h}): 74 \mathrm{mg}$, 黄色固体, 产率 78\%. m.p. $154 \sim 155{ }^{\circ} \mathrm{C} ;{ }^{1} \mathrm{H}$ NMR (400 MHz, $\left.\mathrm{CDCl}_{3}\right) \delta$ : $8.16(\mathrm{~d}, J=8.0 \mathrm{~Hz}, 2 \mathrm{H}), 7.87(\mathrm{~s}, 1 \mathrm{H}), 7.64 \sim 7.57(\mathrm{~m}, 5 \mathrm{H})$, $7.37 \sim 7.33(\mathrm{~m}, 5 \mathrm{H}), 7.10(\mathrm{~d}, J=12.0 \mathrm{~Hz}, 2 \mathrm{H}) ;{ }^{13} \mathrm{C} \mathrm{NMR}$ $\left(101 \mathrm{MHz}, \mathrm{CDCl}_{3}\right) \delta: 149.4,148.7,140.4,137.4,131.8$, 129.9, 129.9, 129.2, 128.9, 128.4, 127.1, 126.1, 112.0; IR (ATR) $v: 3318,1597,1612,1499,1311,1265,1092,835$, $773 \mathrm{~cm}^{-1}$; HRMS (ESI) calcd for $\mathrm{C}_{19} \mathrm{H}_{16} \mathrm{~N}_{3} \mathrm{O}_{2}[\mathrm{M}+\mathrm{H}]^{+}$ 318.1237, found 318.1239.

二苯甲酮-1-菜腙 $(\mathbf{3 i}): 72 \mathrm{mg}$, 黄色固体, 产率 74\%. m.p. $155 \sim 107{ }^{\circ} \mathrm{C}$ (lit. ${ }^{[12 \mathrm{e}]}$ m.p. $155 \sim 156{ }^{\circ} \mathrm{C}$ ). ${ }^{1} \mathrm{H}$ NMR $\left(400 \mathrm{MHz}, \mathrm{CDCl}_{3}\right) \delta: 8.19(\mathrm{~s}, 1 \mathrm{H}), 7.78(\mathrm{~d}, J=8.0 \mathrm{~Hz}$, 2H), 7.67 (dd, $J=8.0,8.0 \mathrm{~Hz}, 4 \mathrm{H}) 7.59$ (t, $J=8.0 \mathrm{~Hz}, 1 \mathrm{H})$, $7.47(\mathrm{dd}, J=8.0,8.0 \mathrm{~Hz}, 3 \mathrm{H}), 7.42 \sim 7.28(\mathrm{~m}, 6 \mathrm{H}), 7.24(\mathrm{~d}$, $J=12.0 \mathrm{~Hz}, 1 \mathrm{H}) ;{ }^{13} \mathrm{C}$ NMR $\left(101 \mathrm{MHz}, \mathrm{CDCl}_{3}\right) \delta: 146.4$, $139.3,138.2,134.2,132.9,129.9,129.5,129.1,128.8$, $128.3,128.3,126.8,126.7,125.6,125.0,121.9,119.9$, 119.0, 108.1.

二苯甲酮-2-氟苯腙 $(\mathbf{3 j}): 51 \mathrm{mg}$, 黄色油状物, 产率 59\%. ${ }^{1} \mathrm{H}$ NMR (400 MHz, $\left.\mathrm{CDCl}_{3}\right) \delta: 7.73(\mathrm{t}, J=8.0 \mathrm{~Hz}$, $1 \mathrm{H}), 7.66(\mathrm{~s}, 1 \mathrm{H}), 7.59$ (t, $J=8.0 \mathrm{~Hz}, 4 \mathrm{H}), 7.52$ (t, $J=8.0$ $\mathrm{Hz}, 1 \mathrm{H}), 7.33$ (t, $J=8.0 \mathrm{~Hz}, 5 \mathrm{H}), 7.12$ (t, $J=4.0 \mathrm{~Hz}, 1 \mathrm{H}$ ), $6.94(\mathrm{q}, J=8.0 \mathrm{~Hz}, 1 \mathrm{H}), 6.75(\mathrm{~d}, J=8.0 \mathrm{~Hz}, 1 \mathrm{H}) ;{ }^{13} \mathrm{C}$ NMR (101 MHz, $\left.\mathrm{CDCl}_{3}\right) \delta: 149.8(\mathrm{~d}, J=238 \mathrm{~Hz}), 146.3$, 138.2, $133.1(\mathrm{~d}, J=9 \mathrm{~Hz}), 132.4,129.8,129.5,128.9$, 128.3, 128.2, 126.7, $124.8(\mathrm{~d}, J=3 \mathrm{~Hz}), 119.4(\mathrm{~d}, J=6$ $\mathrm{Hz}), 114.7$ (d, $J=17 \mathrm{~Hz}), 114.5$ (d, $J=2 \mathrm{~Hz}) ;{ }^{19} \mathrm{~F}$ NMR $\left(376 \mathrm{MHz}, \mathrm{CDCl}_{3}\right.$ ) $\delta:-137.69$; IR (ATR) v: 3351, 1622, $1288,1256,1190,815,746 \mathrm{~cm}^{-1}$; HRMS (ESI) calcd for $\mathrm{C}_{19} \mathrm{H}_{16} \mathrm{FN}_{2}[\mathrm{M}+\mathrm{H}]^{+}$291.1292, found 291.1293.

二苯甲酮-3,5-二氟苯腙 $(\mathbf{3 k}): 76 \mathrm{mg}$, 黄色油状物,
产率 $82 \%$. ${ }^{1} \mathrm{H}$ NMR $\left(400 \mathrm{MHz}, \mathrm{CDCl}_{3}\right) \delta: 7.61 \sim 7.52(\mathrm{~m}$, $6 \mathrm{H}), 7.32(\mathrm{~s}, 1 \mathrm{H}), 6.60(\mathrm{~d}, J=12 \mathrm{~Hz}, 2 \mathrm{H}), 6.26$ (t, $J=8 \mathrm{~Hz}$, $1 \mathrm{H}) ;{ }^{13} \mathrm{C}$ NMR (101 MHz, $\left.\mathrm{CDCl}_{3}\right) \delta: 163.9$ (dd, $J=243$, $15 \mathrm{~Hz}), 146.9$ (t, $J=13 \mathrm{~Hz}), 146.2,137.7,132.3,129.8$, 129.6, 128.9, 128.7, 128.3, 126.8, 96.0 (d, $J=29 \mathrm{~Hz}), 94.9$ (t, $J=26 \mathrm{~Hz}) ;{ }^{19} \mathrm{~F}$ NMR $\left(376 \mathrm{MHz}, \mathrm{CDCl}_{3}\right) \delta$ : -109.72 ; IR (ATR) $v: 3398,1635,1475,1269,1247,1058,786 \mathrm{~cm}^{-}$ ${ }^{1}$; HRMS (ESI) calcd for $\mathrm{C}_{19} \mathrm{H}_{15} \mathrm{~F}_{2} \mathrm{~N}_{2}[\mathrm{M}+\mathrm{H}]^{+} 309.1197$, found 309.1195 .

二苯甲酮-4-甲氧羰基苯腙(黄色油状物): $41 \mathrm{mg}$, 黄 色油状物, 产率 $41 \% .{ }^{1} \mathrm{H}$ NMR (400 MHz, $\mathrm{CDCl}_{3}$ ) $\delta: 7.94$ $(\mathrm{d}, J=28.0 \mathrm{~Hz}, 2 \mathrm{H}), 7.71(\mathrm{~s}, 1 \mathrm{H}), 7.60 \sim 7.54(\mathrm{~m}, 5 \mathrm{H})$, 7.33 (s, 5H), 7.08 (d, $J=8.0 \mathrm{~Hz}, 2 \mathrm{H}), 3.87(\mathrm{~s}, 3 \mathrm{H}) ;{ }^{13} \mathrm{C}$ NMR (101 MHz, $\left.\mathrm{CDCl}_{3}\right) \delta: 167.1,148.1,146.5,137.8$, $132.3,131.4,129.8,129.6,129.0,128.6,128.3,126.8$, 121.3, 112.1, 51.7; IR (ATR) v: 3259, 1698, 1599, 1277, 1261, 1173, 842, $822 \mathrm{~cm}^{-1}$; HRMS (ESI) calcd for $\mathrm{C}_{21} \mathrm{H}_{19} \mathrm{~N}_{2} \mathrm{O}_{2}[\mathrm{M}+\mathrm{H}]^{+}$331.1441, found 331.1445.

二苯甲酮-3-三氟甲基苯腙(3m): $56 \mathrm{mg}$, 黄色油状 物, 产率 55\%. ${ }^{1} \mathrm{H}$ NMR (400 MHz, $\left.\mathrm{CDCl}_{3}\right) \delta: 7.62 \sim 7.48$ (m, $6 \mathrm{H}), 7.34 \sim 7.30(\mathrm{~m}, 7 \mathrm{H}), 7.23(\mathrm{~d}, J=8.0 \mathrm{~Hz}, 1 \mathrm{H})$, $7.08(\mathrm{~d}, J=8.0 \mathrm{~Hz}, 1 \mathrm{H}) ;{ }^{13} \mathrm{C}$ NMR $\left(101 \mathrm{MHz}\right.$, DMSO- $\left.d_{6}\right)$ $\delta: 146.6,145.2,139.0,133.5,130.4,130.1$ (q, $J=31 \mathrm{~Hz})$, $129.9,129.6,129.5,128.8,128.6,126.7,124.9$ (q, $J=270$ $\mathrm{Hz}), 116.9,115.5$ (q, $J=4 \mathrm{~Hz}), 109.9$ (q, $J=4 \mathrm{~Hz}) ;{ }^{19} \mathrm{~F}$ NMR (376 MHz, $\left.\mathrm{CDCl}_{3}\right) \delta:-62.73$; IR(ATR) v: 3336, $1615,1332,1278,1126,805,764,701 \mathrm{~cm}^{-1}$; HRMS (ESI) calcd for $\mathrm{C}_{20} \mathrm{H}_{16} \mathrm{~F}_{3} \mathrm{~N}_{2}[\mathrm{M}+\mathrm{H}]^{+}$341.1260, found 341.1265.

三二苯甲酮-1,3,5-三苯腙(3n): $121 \mathrm{mg}$, 黄色油状 物, 产率 $61 \% .{ }^{1} \mathrm{H}$ NMR $\left(400 \mathrm{MHz}, \mathrm{CDCl}_{3}\right) \delta: 7.60 \sim 7.50$ (m, 5H), $7.46(\mathrm{~s}, 3 \mathrm{H}), 7.35 \sim 7.29(\mathrm{~m}, 4 \mathrm{H}), 6.62(\mathrm{~s}, 3 \mathrm{H})$; ${ }^{13} \mathrm{C} \mathrm{NMR}\left(101 \mathrm{MHz}, \mathrm{CDCl}_{3}\right) \delta: 146.3,145.1,138.1,132.6$, 129.8, 129.4, 129.1, 128.2, 126.6, 105.0, 95.4; IR (ATR) $v$ : 3455, 1604, 1228, $827 \mathrm{~cm}^{-1}$; HRMS (ESI) calcd for $\mathrm{C}_{45} \mathrm{H}_{37} \mathrm{~N}_{6}[\mathrm{M}+\mathrm{H}]^{+}$661.3074, found 661.3072.

二苯甲酮-4-三氟甲基苯腙(3o) ${ }^{[12 a, 17]}: 70 \mathrm{mg}$, 黄色 固体, 产率 69\%. m.p. 93 95 ${ }^{\circ} \mathrm{C}\left(\right.$ lit. ${ }^{[12 a]}$ m.p. 85 86); ${ }^{1} \mathrm{H}$ NMR $\left(400 \mathrm{MHz}, \mathrm{CDCl}_{3}\right) \delta: 7.81(\mathrm{~d}, J=8.0 \mathrm{~Hz}, 1 \mathrm{H})$, $7.64 \sim 7.46(\mathrm{~m}, 8 \mathrm{H}), 7.33(\mathrm{~d}, J=8.0 \mathrm{~Hz}, 4 \mathrm{H}), 7.12(\mathrm{~d}, J=$ $8.0 \mathrm{~Hz}, 2 \mathrm{H}) ;{ }^{13} \mathrm{C} \mathrm{NMR}\left(101 \mathrm{MHz}, \mathrm{CDCl}_{3}\right) \delta: 147.1,146.2$, $137.9,137.6,132.4,132.3,130.1,129.8,129.6,129.0$, 128.6, 128.3, 126.8, 126.6 (q, $J=4 \mathrm{~Hz}), 121.6$ (q, $J=33$ $\mathrm{Hz}), 112.4 ;{ }^{19} \mathrm{~F}$ NMR $\left(376 \mathrm{MHz}, \mathrm{DMSO}-d_{6}\right) \delta$ : -59.37 .

\section{2 .2 芳基苯硼酸的反应}

向 $25 \mathrm{~mL}$ 的反应管中加入 $0.15 \mathrm{mmol}$ 芳基硼酸、 
$0.225 \mathrm{mmol}$ 二苯甲酮腙、 $0.225 \mathrm{mmol}$ 醋酸铜、 $0.30 \mathrm{mmol}$ 吡啶 $-N$-氧化物和 $100 \mathrm{mg}$ 分子篎, 用移液枪加入 $1.0 \mathrm{~mL}$ 二氯甲烷. 反应管用橡皮塞封口, 在室温下搅拌 $24 \mathrm{~h}$, 反应完毕后, 过滤反应混合物, 并用乙酸乙酯洗涤滤饼, 旋蒸滤液除去溶剂. 粗产物经柱层析纯化[洗脱剂: 石 油醚/乙酸乙酯 $(V: V=100: 1)]$ 得到相应的产物.

二苯甲酮一苯腙 $(\mathbf{3 a})^{[12 \mathrm{e}]}$ : $33 \mathrm{mg}$, 黄色固体, 产率 $80 \%$.

二苯甲酮-3-甲基苯腙 $(\mathbf{5 b})^{[12 \mathrm{e}]}$ : $18 \mathrm{mg}$, 黄色油状物, 产率 42\%. ${ }^{1} \mathrm{H} \mathrm{NMR}\left(400 \mathrm{MHz}, \mathrm{CDCl}_{3}\right) \delta: 7.80(\mathrm{~d}, J=8.0$ $\mathrm{Hz}, 1 \mathrm{H}), 7.57$ (t, $J=8.0 \mathrm{~Hz}, 4 \mathrm{H}), 7.49$ (q, $J=8.0 \mathrm{~Hz}, 2 \mathrm{H})$, $7.33 \sim 7.29(\mathrm{~m}, 4 \mathrm{H}), 7.12(\mathrm{t}, J=8.0 \mathrm{~Hz}, 1 \mathrm{H}), 6.89(\mathrm{t}, J=$ $8.0 \mathrm{~Hz}, 2 \mathrm{H}), 6.66(\mathrm{~d}, J=8.0 \mathrm{~Hz}, 1 \mathrm{H}), 2.31(\mathrm{~s}, 3 \mathrm{H}) ;{ }^{13} \mathrm{C}$ NMR (101 MHz, $\left.\mathrm{CDCl}_{3}\right) \delta: 144.6,144.0,139.1,138.5$, $129.7,129.2,129.2,128.2,126.5,121.0,113.5,110.2$, 21.6.

二苯甲酮-4-三氟甲氧基苯腙 $(5 c): 27 \mathrm{mg}$, 黄色油状 物, 产率 51\%. ${ }^{1} \mathrm{H} \mathrm{NMR}\left(400 \mathrm{MHz}, \mathrm{CDCl}_{3}\right) \delta: 7.80$ (d, $J=8.0 \mathrm{~Hz}, 2 \mathrm{H}), 7.59$ (t, $J=8.0 \mathrm{~Hz}, 3 \mathrm{H}), 7.54$ (d, $J=8.0$ $\mathrm{Hz}, 1 \mathrm{H}), 7.48(\mathrm{t}, J=8.0 \mathrm{~Hz}, 3 \mathrm{H}), 7.34 \sim 7.31(\mathrm{~m}, 4 \mathrm{H}), 7.08$ $(\mathrm{q}, J=8.0 \mathrm{~Hz}, 2 \mathrm{H}) ;{ }^{13} \mathrm{C} \mathrm{NMR}\left(101 \mathrm{MHz}, \mathrm{CDCl}_{3}\right) \delta: 143.4$, $137.6,132.4,130.1,129.8,129.4,129.0,128.3,128.2$, 126.6, 122.3, 116.2, 113.5; ${ }^{19} \mathrm{~F} \quad \mathrm{NMR} \quad(376 \mathrm{MHz}$, DMSO- $\left.d_{6}\right) \delta$ : 57.28 ; IR (ATR) $v: 3339,1620,1318$, 1254, 1209, 827, $786 \mathrm{~cm}^{-1}$; HRMS (ESI) calcd for $\mathrm{C}_{20} \mathrm{H}_{16} \mathrm{~F}_{3} \mathrm{~N}_{2} \mathrm{O}[\mathrm{M}+\mathrm{H}]^{+}$357.1254, found 357.1256.

二苯甲酮-3-甲氧基苯腙(3c) ${ }^{[12 \mathrm{e}]}$ : $20 \mathrm{mg}$, 黄色固体, 产率 $45 \%$.

二苯甲酮-4-氯苯腙(5e)：33 mg, 黄色固体, 产率 71\%. m.p. $115 \sim 117{ }^{\circ} \mathrm{C}$ (lit. ${ }^{[12 \mathrm{e}]}$ m.p. $116 \sim 117{ }^{\circ} \mathrm{C}$ ). ${ }^{1} \mathrm{H}$ NMR (400 MHz, $\left.\mathrm{CDCl}_{3}\right) \delta: 7.58 \sim 7.46(\mathrm{~m}, 6 \mathrm{H}), 7.32$ (q, $J=8.0 \mathrm{~Hz}, 5 \mathrm{H}), 7.19$ (d, $J=8.0 \mathrm{~Hz}, 2 \mathrm{H}), 7.00$ (t, $J=12.0$ $\mathrm{Hz}, 2 \mathrm{H}) ;{ }^{13} \mathrm{C} \mathrm{NMR}\left(101 \mathrm{MHz}, \mathrm{CDCl}_{3}\right) \delta: 144.9,143.3$, $137.6,132.5,130.1,129.8,129.1,129.1,128.3,128.2$, 126.6, 124.6, 114.1.

二苯甲酮-4-溴苯腙 $(\mathbf{5 f})^{[12 \mathrm{e}]}: 39 \mathrm{mg}$, 黄色油状物, 产 率 75\%. ${ }^{1} \mathrm{H}$ NMR $\left(400 \mathrm{MHz}, \mathrm{CDCl}_{3}\right) \delta: 7.60 \sim 7.52(\mathrm{~m}$, 5H), $7.46(\mathrm{~s}, 1 \mathrm{H}), 7.32$ (d, $J=8.0 \mathrm{~Hz}, 7 \mathrm{H}), 6.96(\mathrm{~d}, J=8.0$ $\mathrm{Hz}, 2 \mathrm{H}) ;{ }^{13} \mathrm{C} \mathrm{NMR}\left(101 \mathrm{MHz}, \mathrm{CDCl}_{3}\right) \delta: 145.0,143.7$, $138.1,132.5,132.0,130.1,129.8,129.1,128.3,128.2$, $126.6,114.5,111.8$.

二苯甲酮-3-氟苯腙(3d): $22 \mathrm{mg}$, 黄色油状物, 产率 $50 \%$.

\section{2 .3 二苯甲酮一苯腙水解制备苯肼}

向 $25 \mathrm{~mL}$ 反应管中加入 $0.2 \mathrm{mmol} \mathrm{3a}, 2.0 \mathrm{~mL}$ 乙醇/
浓盐酸混合液 $(V: V=10 ： 1)$, 混合物在室温下搅拌 24 $h$, 反应完成后过滤得到粗产物，二氯甲烷洗涤可得到 纯化的盐酸苯肼(3aa), 白色固体，产率 56\%. m.p. 223 $224{ }^{\circ} \mathrm{C}$ (lit. ${ }^{[12 \mathrm{e}]}$ m.p. $\left.222 \sim 224{ }^{\circ} \mathrm{C}\right) .{ }^{1} \mathrm{H}$ NMR (400 MHz, DMSO-d $\left.d_{6}\right) \delta$ : $10.32(\mathrm{~d}, J=32.0 \mathrm{~Hz}, 3 \mathrm{H}), 7.28$ (t, $J=8.0$ $\mathrm{Hz}, 2 \mathrm{H}), 7.01$ (d, $J=8.0 \mathrm{~Hz}, 2 \mathrm{H}), 6.94$ (d, $J=8.0 \mathrm{~Hz}, 1 \mathrm{H})$; ${ }^{13} \mathrm{C}$ NMR (101 MHz, DMSO- $\left.d_{6}\right) \delta: 146.1,129.4,121.8$, 115.0 .

辅助材料(Supporting Information) 化合物 $\mathbf{3 a} \sim \mathbf{3 0}$, 5b, 5c, 5e, 5f, 3aa 的 ${ }^{1} \mathrm{H}$ NMR 和 ${ }^{13} \mathrm{C}$ NMR 谱图. 这些材 料可以免费从本刊网站(http://sioc-journal.cn/)上下载.

\section{References}

[1] Humphrey, G. R.; Kuethe, J. T. Chem. Rev. 2006, 106, 2875.

[2] Fustero, S.; Sánchez-Roselló, M.; Barrio, P.; Simón-Fuentes, A. Chem. Rev. 2011, 111, 6984.

[3] (a) Xu, Z.; Wang, D.-S.; Yu, X.; Yang, Y.; Wang, D. Adv. Synth. Catal. 2017, 359, 3332.

(b) Yang, Y.; Qin, A.; Zhao, K.; Wang, D.; Shi, X. Adv. Synth. Catal. 2016, 358, 1433.

(c) Hu, X.; Yang, B.; Yao, W.; Wang, D. Chin. J. Org. Chem. 2018 38, 3296 (in Chinese).

(胡昕宇，杨伯斌，姚玮，王大伟，有机化学, 2018, 38, 3296.)

(d) Huang, R.; Yang, Y.; Wang, D.-S.; Zhang, L.; Wang, D. Org. Chem. Front. 2018, 5, 203.

(e) Wu, Q.; Pan, L.; Du, G.; Zhang, C.; Wang, D. Org. Chem. Front 2018, $5,2668$.

[4] (a) Wang, L.; Woods, K. W.; Li, Q.; Barr, K. J.; McCroskey, R. W.; Hannick, S. M.; Gherke, L.; Credo, R. B.; Hui, Y.-H.; Marsh, K.; Warner, R.; Lee, J. Y.; Zielinski-Mozng, N.; Frost, D.; Rosenberg, S. H.; Sham, H. L. J. Med. Chem. 2002, 45, 169.

(b) Köhling, P.; Schmidt, A. M.; Eilbracht, P. Org. Lett. 2003, 5, 3213 .

(c) Kuethe, J. T.; Wong, A.; Qu, C.; Smitrovich, J.; Davies, I. W.; Hughes, D. L. J. Org. Chem. 2005, 70, 2555.

(d) Jukes, R. T. F.; Bozic, B.; Hartl, F.; Belser, P.; De Cola, L. Inorg Chem. 2006, 45, 8326 .

(e) Zhang, T.; Bao, W. J. Org. Chem. 2013, 78, 1317.

(f) Dubost, E.; Stiebing, S.; Ferrary, T.; Cailly, T.; Fabis, F.; Collot, V. Tetrahedron 2014, 70, 8413.

(g) Wei, W.; Wang, Z.; Yang, X.; Yu, W.; Chang, J. Adv. Synth. Catal. 2017, 359, 3378.

[5] (a) Schlummer, B.; Scholz, U. Adv. Synth. Catal. 2004, 346, 1599. (b) Hartwig, J. F. Acc. Chem. Res. 2008, 41, 1534.

(c) Ruiz-Castillo, P.; Buchwald, S. L. Chem. Rev. 2016, 116, 12564.

[6] (a) Hartwig, J. F. Angew. Chem., Int. Ed. 1998, 37, 2090.

(b) Wagaw, S.; Yang, B. H.; Buchwald, S. L. J. Am. Chem. Soc. 1999, 121, 10251 .

(c) Mauger, C.; Mignani, G. Adv. Synth. Catal. 2005, 347, 773

[7] (a) Wang, Z.; Skerlj, R. T.; Bridger, G. J. Tetrahedron Lett. 1999 40,3543 .

(b) Arterburn, J. B.; Rao, K. V.; Ramdas, R.; Dible, B. R. Org. Lett. 2001, 3, 1351 .

(c) Lim, Y.-K.; Lee, K.-S.; Cho, C.-G. Org. Lett. 2003, 5, 979.

(d) Halland, N.; Nazaré, M.; Alonso, J.; R'Kyek, O.; Lindenschmidt, A. Chem. Commun. 2011, 47, 1042.

[8] (a) Lundgren, R. J.; Stradiotto, M. Angew. Chem., Int. Ed. 2010, 49, 8686.

(b) DeAngelis, A.; Wang, D.-H.; Buchwald, S. L. Angew. Chem., Int. Ed. 2013, 52, 3434. 
[9] Ley, S. V.; Thomas, A. W. Angew. Chem., Int. Ed. 2003, 42, 5400. (b) Bhunia, S.; Pawar, G. G.; Kumar, S. V.; Jiang, Y.; Ma, D. Angew. Chem., Int. Ed. 2017, 56, 16136.

[10] (a) Wolter, M.; Klapars, A.; Buchwald, S. L. Org. Lett. 2001, 3, 3803 .

(b) Lam, M. S.; Lee, H. W.; Chan, A. S. C.; Kwong, F. Y. Tetrahedron Lett. 2008, 49, 6192.

(c) Jiang, L.; Lu, X.; Zhang, H.; Jiang, Y.; Ma, D. J. Org. Chem. 2009, 74, 4542.

(d) Xiong, X.; Jiang, Y.; Ma, D. Org. Lett. 2012, 14, 2552.

[11] Chen, J.; Zhang, Y.; Hao, W.; Zhang, R.; Yi, F. Tetrahedron 2013, 69,613 .

[12] (a) Wagaw, S.; Yang, B. H.; Buchwald, S. L. J. Am. Chem. Soc. 1998, 120, 6621.

(b) Mauger, C. C.; Mignani, G. A. Org. Process Res. Dev. 2004, 8, 1065 .

(c) Lefebvre, V.; Cailly, T.; Fabis, F.; Rault, S. J. Org. Chem. 2010, 75,2730 .

(d) Goodyear, A.; Linghu, X.; Bishop, B.; Chen, C.; Cleator, E.; McLaughlin, M.; Sheen, F. J.; Stewart, G. W.; Xu, Y.; Yin, J. Org. Process Res. Dev. 2012, 16, 605.

(e) Wu, W.; Fan, X.-H.; Zhang, L.-P.; Yang, L.-M. RSC Adv. 2014, 4, 3364 .

(f) Chen, Z.; Huo, Y.; An, P.; Wang, X.; Song, C.; Ma, Y. Org. Chem. Front. 2016, 3, 1725.
[13] (a) Shen, Q.; Shekhar, S.; Stambuli, J. P.; Hartwig, J. F. Angew. Chem., Int. Ed. 2005, 44, 1371.

(b) Tardiff, B. J.; Stradiotto, M. Eur. J. Org. Chem. 2012, 2012, 3972 .

[14] (a) Qiao, J.; Lam, P. Synthesis 2010, 829.

(b) Sanjeeva Rao, K.; Wu, T.-S. Tetrahedron 2012, 68, 7735.

(c) Corbet, J.-P.; Mignani, G. Chem. Rev. 2006, 106, 2651.

(d) Allen, S. E.; Walvoord, R. R.; Padilla-Salinas, R.; Kozlowski, M. C. Chem. Rev. 2013, 113, 6234.

(e) Ma, X. P.; Liu, F. P.; Mo, D. L. Chin. J. Org. Chem. 2017, 37, 1069 (in Chinese) (马小盼, 刘凤萍, 莫冬亮, 有机化学, 2017, 37, 1069.)

[15] (a) Liu, S.; Zu, W.; Zhang, J.; Xu, L. Org. Biomol. Chem. 2017, 15, 9288.

(b) Liu, S.; Xu, L. Asian J. Org. Chem. 2018, 7, 1856.

(c) Zu, W.; Liu, S.; Jia, X.; Xu, L. Org. Chem. Front. 2019, 6, 1356.

(d) Liang, X.; Xu, L.; Li, C.; Jia, X.; Wei, Y. Tetrahedron 2019, 75, 721 .

(e) Liu, S.; Xu, L.; Wei, Y. J. Org. Chem. 2019, 84, 1596.

[16] (a) Surry, D. S.; Buchwald, S. L. Angew. Chem., Int. Ed. 2008, 47, 6338 .

(b) Surry, D. S.; Buchwald, S. L. Chem. Sci. 2011, 2, 27.

[17] Li, X.; He, L.; Chen, H.; Wu, W.; Jiang, H. J. Org. Chem. 2013, 78, 3636.

(Zhao, C.) 